\title{
Path Feasibility Analysis for String-Manipulating Programs
}

\author{
Nikolaj Bjørner ${ }^{1}$, Nikolai Tillmann ${ }^{1}$, and Andrei Voronkov ${ }^{2}$ \\ 1 Microsoft Research \\ $\frac{\text { http: / / research.microsoft.com }}{2 \text { University of Manchester }}$ \\ http://www. voronkov.com
}

\begin{abstract}
We discuss the problem of path feasibility for programs manipulating strings using a collection of standard string library functions. We prove results on the complexity of this problem, including its undecidability in the general case and decidability of some special cases. In the context of test-case generation, we are interested in an efficient finite model finding method for string constraints. To this end we develop a two-tier finite model finding procedure. First, an integer abstraction of string constraints are passed to an SMT (Satisfiability Modulo Theories) solver. The abstraction is either unsatisfiable, or the solver produces a model that fixes lengths of enough strings to reduce the entire problem to be finite domain. The resulting fixed-length string constraints are then solved in a second phase. We implemented the procedure in a symbolic execution framework, report on the encouraging results and discuss directions for improving the method further.
\end{abstract}

\section{Introduction}

Dynamic symbolic execution [8]3/14]16] has recently gained attention in the context of test-case generation. It extends static symbolic execution [11] by collecting symbolic constraints from concrete execution traces obtained by monitoring the executed instructions. In order to explore a different execution path it suffices to modify one of the extracted symbolic traces by selecting and negating a branch condition, which we call flipping a branch. Then a constraint solver is used to provide a satisfying assignment to the modified path condition.

Strings form a fundamental data type found in most if not all general purpose programming languages. Strings may be represented in various ways, such as a pointer to a 0-terminated array of characters (in C), as an array object with an explicit length (in Java and C\#), or even as a singly linked list (in Haskell). Programs that manipulate strings can often abstract from the representation and use a set of library routines to perform common functions on strings, such as converting characters to strings, finding characters and extracting substrings.

The problem. String library routines are themselves implemented as programs, so dynamic symbolic execution can apply to string routines by exploring the underlying programs and solving constraints on the data types used in these programs. There is 
an inherent overhead of this approach, as the general dynamic symbolic exploration engine has to search the state space of the string library routines for solutions to string constraints. We can take advantage of the fact that path constraints from common string library routines have a mathematical abstraction rooted in word equations. This suggests that we may work at the level of abstract strings and treat calls to the string library functions as operations in a theory of strings. As we will see, the full set of constraints that can be created from common string functions do not fall in a decidable class. On the other hand, our objective is really to find small strings that can be supplied as unit tests, so an incremental small and finite model-finding routine provides the right match.

The main existing way of handling strings in symbolic test-case generation tools is to not handle them specially. For example in the current release of Pex [16], strings are represented as arrays and string library routines are explored like any other procedure. As a result, simple calls to library functions become programs containing loops. Summaries [7] provide one additional layer on top of the string procedures to allow the search on feasible paths to coalesce several traversals of the same procedure body.

Example 1. Consider the program shown in Figure 1 This program checks whether the input string $\mathrm{s}$ is a URL encoding a query about EasyChair to either Microsoft Live Search or Google. Essentially, such a string must start with "http://" followed by a domain of one of the search engines, followed by a " $/$, and after this " $/$, it should contain a substring "EasyChair" and not contain other" $/$ ".

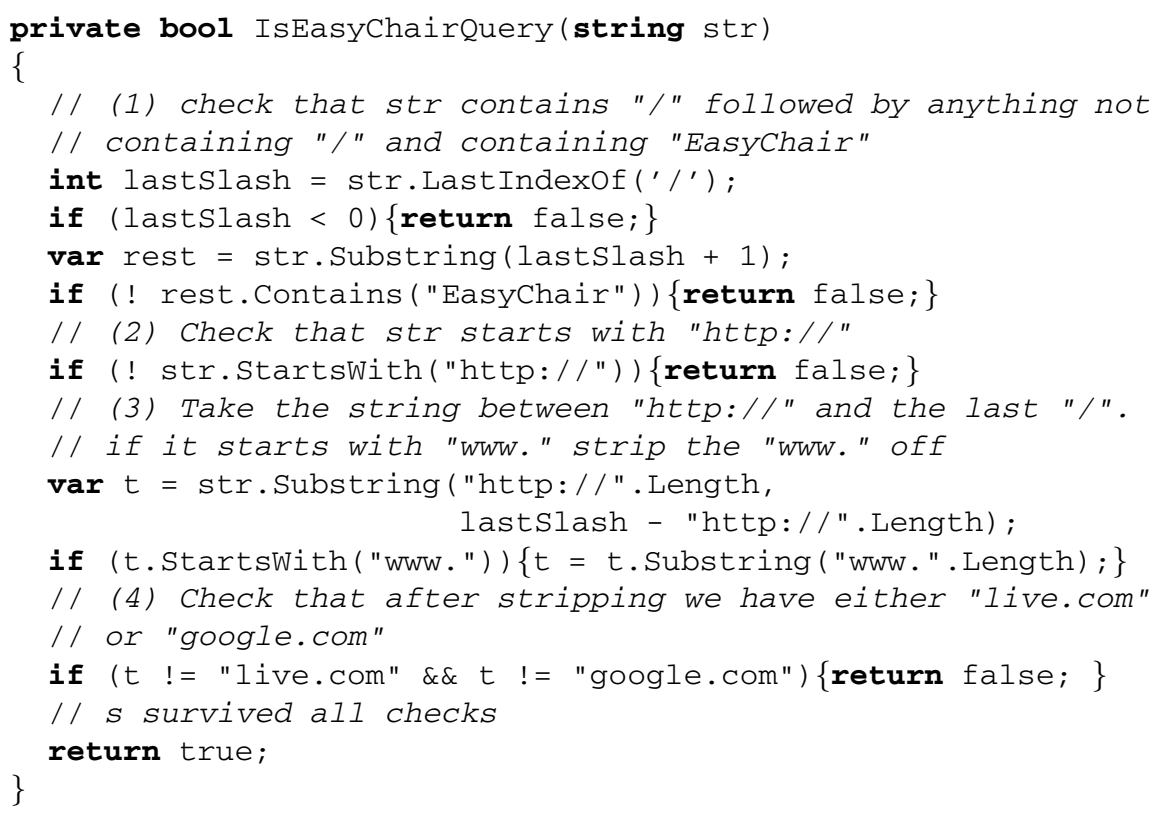

Fig. 1. The EasyChair Query program

We will use this program as the running example. Consider the following path in the program, where queries are denoted by “?”. 


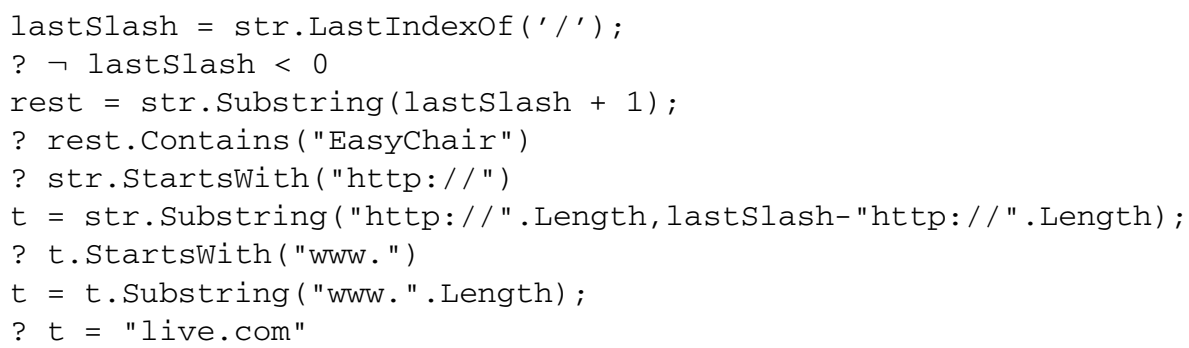

We are interested in checking feasibility of this path, that is, finding an input string str so that the program run with this string as an input will follow this path.

The rest of this paper is organized as follows. Section 2 defines path feasibility in the context of constraints from basic .NET string library functions and defines a first-order string library language corresponding to these library functions. The resulting constraints for all but one library function are compiled into the core language as outlined in Section 3. Section 4 discusses the decidability of the library language. One fragment is equivalent to a long standing open problem in word equations, another fragment is shown undecidable. This confirms the complexity of the path feasibility problem for string library functions. We also show that a so-called fixed-length fragment is decidable: this fact is used in our implementation. We point out that two other decidable fragments can be obtained using results from word equations and automatic structures. Section 5 outlines our incremental procedure for enumerating solutions to core language constraints. Sections 6 and 7 describe an implementation and evaluation.

\section{Path Feasibility and String Constraints}

In this section we define a language for representing the path feasibility problem as a constraint satisfaction problem.

The String Library Language. In this section we will introduce a string library language $\mathcal{L} \mathcal{L}$. This language is a first-order language for describing constraints involving string library functions. The string library we are interested in is the . NET String library, however, we believe that other string libraries can be captured by similar languages and processed using the methods described in this paper.

Let $\mathbb{C}$ be a finite set of characters and $\mathbb{S}$ the set of all strings built using these characters. By $\mathbb{I}$ we denote the set of all integers. The string library language $\mathcal{L} \mathcal{L}$ is a many-sorted first-order language defined as follows. The language has the following sorts: the character sort $\mathcal{C}$, the string sort $\mathcal{S}$, the integer sort $\mathcal{I}$ and the Boolean sort $\mathcal{B}$. It also contains a countably infinite number of variables of each sort.

The language $\mathcal{L} \mathcal{L}$ contains constants denoting all integers, characters and strings, and some functions on strings and integers. Table 1 contains a description of a representative subset of the . NET string library. Positions in strings are number from 0 so the character at the position 0 in a string is always the first character of the string.

Some functions of the . NET library are overloaded. To resolve ambiguity, we add indexes to the names of these functions. For example, there are six different . NET 
Table 1. String library functions

\begin{tabular}{|c|c|c|}
\hline function & type & meaning \\
\hline $\operatorname{Chars}(s, i)$ & $\mathcal{S} \times \mathcal{I} \rightarrow \mathcal{C}$ & the character at position $i$ in $s$ \\
\hline Compare $\left(s_{1}, s_{2}\right)$ & $\mathcal{S} \times \mathcal{S} \rightarrow \mathcal{I}$ & string comparison \\
\hline Concat $\left(s_{1}, s_{2}\right)$ & $\mathcal{S} \times \mathcal{S} \rightarrow \mathcal{S}$ & string concatenation \\
\hline $\operatorname{Contains}\left(s_{1}, s_{2}\right)$ & $\mathcal{S} \times \mathcal{S} \rightarrow \mathcal{B}$ & true if $s_{2}$ is a substring of $s_{1}$ \\
\hline Equals $\left(s_{1}, s_{2}\right)$ & $\mathcal{S} \times \mathcal{S} \rightarrow \mathcal{B}$ & true if $s_{1}=s_{2}$ \\
\hline $\operatorname{IndexOf}_{4}\left(s_{1}, s_{2}, i\right)$ & $\mathcal{S} \times \mathcal{S} \times \mathcal{I} \rightarrow \mathcal{I}$ & $\begin{array}{l}\text { the index of the first occurrence of } s_{2} \text { in } s_{1} \text { starting at po- } \\
\text { sition } i \text { or }-1 \text { if not found }\end{array}$ \\
\hline LastIndexOf $f_{1}(s, c)$ & $\mathcal{S} \times \mathcal{C} \rightarrow \mathcal{I}$ & the index of the last occurrence of $c$ in $s$ or -1 if not found \\
\hline Length $(s)$ & $\mathcal{S} \rightarrow \mathcal{I}$ & the length of $s$ \\
\hline $\operatorname{Replace}_{2}\left(s_{1}, s_{2}, s_{3}\right)$ & $\mathcal{S} \times \mathcal{S} \times \mathcal{S} \rightarrow \mathcal{S}$ & replace all occurrences of $s_{2}$ in $s_{1}$ with $s_{3}$ \\
\hline $\operatorname{StartsWith}\left(s_{1}, s_{2}\right)$ & $\mathcal{S} \times \mathcal{S} \rightarrow \mathcal{B}$ & true if $s_{1}$ starts with $s_{2}$ \\
\hline Substring $_{1}(s, i)$ & $\mathcal{S} \times \mathcal{I} \rightarrow \mathcal{S}$ & substring of $s$ starting at position $i$ \\
\hline Substring $_{2}\left(s, i_{1}, i_{2}\right)$ & $\mathcal{S} \times \mathcal{I} \times \mathcal{I} \rightarrow \mathcal{S}$ & substring of $s$ starting at position $i_{1}$ and having length $i_{2}$ \\
\hline ToString $(c)$ & $\mathcal{C} \rightarrow \mathcal{S}$ & string consisting of a single character $c$ \\
\hline ToUpper $(s)$ & $\mathcal{S} \rightarrow \mathcal{S}$ & string obtained by converting $s$ to upper case \\
\hline
\end{tabular}

functions having the name Index $O f$. In $\mathcal{L} \mathcal{L}$ we add indexes to function names to distinguish them (cf. IndexOf $f_{4}$ in the table).

To define the semantics of $\mathcal{L} \mathcal{L}$ we introduce a notion of value assignment. A value assignment for this language is a mapping of variables to $\mathbb{C} \cup \mathbb{S} \cup \mathbb{I}$ so that every variable is mapped to an element of the corresponding domain, for example, variables of the sort $\mathcal{I}$ are mapped to integer values. We will now extend value assignments to arbitrary expressions and quantifier-free formulas. To this end we should take care of undefined values since some of the library functions are partial and calling them outside of their domain causes an exception. We will specify the exception conditions later.

Let us introduce a special undefined value $\perp$ and extend value assignments to arbitrary expressions of $\mathcal{L} \mathcal{L}$ as follows. For every value assignment $v$ and expression $e$ different from a variable we define $v(e)$ as follows.

1. If $e$ is a constant, then $v(e)$ is the value of this constant, for example the value of the integer constant 7 is the number 7 .

2. Suppose that $e$ has the form $f\left(e_{1}, \ldots, e_{n}\right)$ where $f$ is a function of $\mathcal{L} \mathcal{L}$ and let $v_{1}=v\left(e_{1}\right), \ldots, v_{n}=v\left(e_{n}\right)$. If for some $i \in\{1, \ldots, n\}$ we have $v_{i}=\perp$, then $v(e)=\perp$. Otherwise, let the $\widehat{f}$ be the function corresponding to $f$ (see Table 1 for the definition of these functions). If $\widehat{f}$ is undefined on $\left(v_{1}, \ldots, v_{n}\right)$, then $v(e)=\perp$, otherwise $v(e)$ if the value $\widehat{f}\left(v_{1}, \ldots, v_{n}\right)$.

If $v(e)=\perp$, we say that $v(e)$ is undefined under the value assignment $v$.

The next step is to define the semantics of formulas. To this end we will use the threevalued logic having the Boolean values true and false and the undefined value $\perp$. In this logic, for example, a disjunction is true if at least one of its members is true; false if all of them are false and undefined otherwise. We omit details due to lack of space. 
Path Feasibility. Suppose that $\pi$ is a path in a program using only assignments and tests. The path feasibility problem is the problem of finding initial values of variables which make the path feasible, i.e., values so that the program execution starting with this assignment satisfies all tests on the paths and raises no exceptions. The path feasibility problem can be reduced to the following constraint satisfaction problem for $\mathcal{L} \mathcal{L}$ :

Given a finite set $\left\{L_{1}, \ldots, L_{n}\right\}$ of literals, find a value assignment $v$ which makes all of these literals true.

Evidently, the path feasibility problem can be reduced in a straightforward way to the constraint satisfaction problem for $\mathcal{L} \mathcal{L}$. We do not give full details here but restrict ourselves to an example.

Example 2. Consider the path from Example 1 This path can be translated to the following constraint.

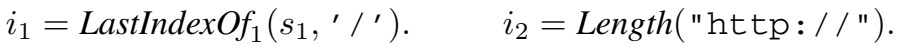

$$
\begin{aligned}
& \neg i_{1}<0 . \quad s_{3}=\operatorname{Substring}_{2}\left(s_{1}, \ell, i_{1}-i_{2}\right) \text {. } \\
& s_{2}=\operatorname{Substring}{ }_{1}\left(s_{1}, i_{1}+1\right) . \quad \operatorname{StartsWith}\left(s_{3}\right. \text {, "Www. " ). }
\end{aligned}
$$

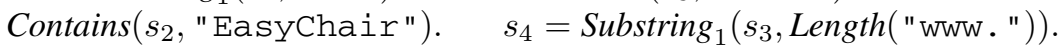

$$
\begin{aligned}
& \text { StartsWith }\left(s_{1}, \text { "http://"). } s_{4}=\right.\text { "live.com". }
\end{aligned}
$$

This constraint is satisfiable if and only if there exists an initial value of the variable $s_{1}$ so that the program will follow the intended path.

\section{The Core String Language}

In this section we will define the so-called core string language $\mathcal{C} \mathcal{L}$. This language contains a smaller number of functions than $\mathcal{L} \mathcal{L}$ but we will be interested in a larger fragment of this language, including propositional connectives and bounded quantifiers of a special form. This language will play the role of an intermediate language between the string library language and an SMT solver. We will start with defining $\mathcal{C} \mathcal{L}$ and giving a translation of $\mathcal{L} \mathcal{L}$ into $\mathcal{C} \mathcal{L}$. This translation will also define the semantics of the $\mathcal{L} \mathcal{L}$ functions in a strict way as opposed to a less formal description in Table 1.

Definition 1 (core string language). For simplicity we will usually say the library language instead of the string library language and the core language instead of the core string language. Likewise, we will simply say library functions and core functions. The core language contains the following functions and predicates:

1. The string functions Length and Chars. We will write $\ell(s)$ instead of Length $(s)$ and $s[i]$ instead of $\operatorname{Chars}(s, i)$.

2. The standard functions and predicates of linear integer arithmetic.

3. Some functions and predicates on characters. We do not specify the set of all these functions but will use two of them (comparison $<$ and ToUpper) later. 
The formulas of the language are defined as follows:

1. Every predicate specified above is a formula;

2. If $F_{1}$ and $F_{2}$ are formulas, then $\neg F_{1}, F_{1} \wedge F_{2}, F_{1} \vee F_{2}$ and $F_{1} \rightarrow F_{2}$ are formulas.

3. If $F$ is a formula, $i$ a variable of the sort $\mathcal{I}$ and $\ell, u$ expressions of the sort $\mathcal{I}$ not containing $i$, then $(\forall i)(\ell \leq i \wedge i \leq u \rightarrow F)$ and $(\exists i)(\ell \leq i \wedge i \leq u \wedge F)$ are formulas. In the following, we will write these instead as: $(\forall i \in[\ell \ldots u]) F$ and $(\exists i \in[\ell \ldots u]) F$ respectively.

Note the following equivalences:

$$
\neg(\forall i \in[\ell \ldots u]) F \equiv(\exists i \in[\ell \ldots u]) \neg F ; \quad \neg(\exists i \in[\ell \ldots u]) F \equiv(\forall i \in[\ell \ldots u]) \neg F .
$$

Table 2. Exception conditions for the library functions

\begin{tabular}{|l|l|}
\hline Function & exception condition \\
\hline Chars $(s, i)$ & $i<0 \vee i \geq \ell(s)$ \\
IndexOf $_{4}\left(s_{1}, s_{2}, i\right)$ & $i<0 \vee \ell\left(s_{1}\right) \geq i$ \\
Replace $_{2}\left(s_{1}, s_{2}, s_{3}\right)$ & $\ell\left(s_{2}\right)=0$ \\
Substring $_{1}(s, i)$ & $i<0 \vee i>\ell(s)$ \\
Substring $_{2}\left(s, i_{1}, i_{2}\right)$ & $i_{1}<0 \vee i_{2}<0 \vee i_{1}+i_{2}>\ell(s)$ \\
\hline
\end{tabular}

Let us now define formally the translation of the library language into the core language. We will do this by first defining exception conditions for all library functions and then the library functions themselves using the core language. The exception con-

ditions are given in Table 2 . Note that $\ell$ is the only non-arithmetical core function used in the exception conditions.

Let us introduce two abbreviations for the core language as follows.

$$
\begin{array}{r}
s_{1}[i \ldots j]=s_{2} \stackrel{\text { def }}{=} \ell\left(s_{2}\right)=j-i+1 \wedge(\forall k \in[i \ldots j])\left(s_{1}[k]=s_{2}[k-i]\right) . \\
s_{1} \sqsubseteq s_{2} \stackrel{\text { def }}{=}\left(\exists i \in\left[0 \ldots \ell\left(s_{2}\right)-\ell\left(s_{1}\right)\right]\right) s_{2}\left[i \ldots i+\ell\left(s_{1}\right)-1\right]=s_{1} .
\end{array}
$$

One can easily show that $s_{1} \sqsubseteq s_{2}$ is equivalent to $\operatorname{Contain}\left(s_{2}, s_{1}\right)$ and to $\exists s_{3} \exists s_{4}\left(s_{3}\right.$. $s_{1} \cdot s_{4}=s_{2}$ ). The difference is that $s_{1} \sqsubseteq s_{2}$ is a formula of the core language.

Let us now give a definition for every library function in the core language. This, together with the definition of exception conditions, also provides a precise semantics for the library functions instead of the less formal explanation given in Table 1 . The definitions of the library functions are given in Table 3 .

We are interested in the following fragment of the core language.

Definition 2. Let $F$ be a formula of either the library or the core language such that (i) the string variables occurring in $F$ are $s_{1}, \ldots, s_{n}$ and (ii) $F$ contains no free occurrences of integer variables. The formula $F$ is said to be a fixed-length formula if it has the form

$$
\ell\left(s_{1}\right)=i_{1} \wedge \ldots \wedge \ell\left(s_{n}\right)=i_{n} \wedge F^{\prime}
$$

where $i_{1}, \ldots, i_{n}$ are concrete integer constants occurring in $F^{\prime}$. The fixed-length fragment of the library (respectively, core) language is the set of all fixed-length formulas of this language. 
Table 3. Definitions of some library predicates

\begin{tabular}{|c|c|}
\hline function/predicate & definition \\
\hline $\operatorname{Chars}(s, i)$ & $s[i]$ \\
\hline Compare $\left(s_{1}, s_{2}\right)=0$ & $s_{1}=s_{2}$ \\
\hline Compare $\left(s_{1}, s_{2}\right)<0$ & $\begin{array}{c}\left(\exists i \in\left[0 \ldots \ell\left(s_{1}\right)-1\right]\right)\left(\ell\left(s_{2}\right)>i \wedge s_{1}[0 \ldots i-1]=s_{2}[0 \ldots i-1] \wedge\right. \\
\left.\left(\ell\left(s_{1}\right)=i \vee s_{1}[i]<s_{2}[i]\right)\right)\end{array}$ \\
\hline Compare $\left(s_{1}, s_{2}\right)>0$ & $\begin{array}{c}\left(\exists i \in\left[0 \ldots \ell\left(s_{2}\right)-1\right]\right)\left(\ell\left(s_{1}\right)>i \wedge s_{1}[0 \ldots i-1]=s_{2}[0 \ldots i-1] \wedge\right. \\
\left.\left(\ell\left(s_{2}\right)=i \vee s_{2}[i]<s_{1}[i]\right)\right)\end{array}$ \\
\hline $\operatorname{Concat}\left(s_{1}, s_{2}\right)$ & $s_{1} \cdot s_{2}$ \\
\hline Contains $\left(s_{1}, s_{2}\right)$ & $s_{2} \sqsubseteq s_{1}$ \\
\hline Equals $\left(s_{1}, s_{2}\right)$ & $s_{1}=s_{2}$ \\
\hline $\operatorname{IndexOf}_{4}\left(s_{1}, s_{2}, i_{1}\right)=i_{0}$ & $\begin{array}{l}\left(i_{0}=-1 \wedge s_{2} \not s_{1}\left[i_{1} \ldots \ell\left(s_{1}\right)-1\right]\right) \vee \\
\left(i_{0} \geq i_{1} \wedge s_{1}\left[i_{0} \ldots i_{0}+\ell\left(s_{2}\right)-1\right]=s_{2} \wedge\right. \\
\left.\quad\left(\forall j \in\left[i_{1} \ldots i_{0}-1\right]\right)\left(s_{1}\left[j \ldots j+\ell\left(s_{2}\right)-1\right] \neq s_{2}\right)\right)\end{array}$ \\
\hline LastIndexOf $f_{1}(s, c)=i$ & $\begin{array}{l}(i=-1 \wedge(\forall j \in[0 \ldots \ell(s)-1]) s[j] \neq c) \vee \\
(i \geq 0 \wedge s[i]=c \wedge(\forall j \in[i+1 \ldots \ell(s)-1]) s[j] \neq c)\end{array}$ \\
\hline Replace $_{2}\left(s_{1}, s_{2}, s_{3}\right)$ & no definition exists \\
\hline $\operatorname{StartsWith}\left(s_{1}, s_{2}\right)$ & $\left(\exists i \in\left[0 \ldots \ell\left(s_{1}\right)-\ell\left(s_{2}\right)\right]\right) s[0 \ldots i-1]=s_{2}$ \\
\hline $\operatorname{Substring}_{1}(s, i)$ & $s[i \ldots \ell(s)-1]$ \\
\hline $\operatorname{Substring}_{2}\left(s, i_{1}, i_{2}\right)$ & $s\left[i_{1} \ldots i_{1}+i_{2}-1\right]$ \\
\hline ToString $(c)=s$ & $\ell(s)=1 \wedge s[0]=c$ \\
\hline ToUpper $\left(s_{1}\right)=s_{2}$ & $\ell\left(s_{2}\right)=\ell\left(s_{1}\right) \wedge\left(\forall i \in\left[0 \ldots \ell\left(s_{1}\right)-1\right]\right) \operatorname{Upper}\left(s_{1}[i], s_{2}[i]\right)$ \\
\hline
\end{tabular}

Theorem 1. The satisfiability problem for the fixed-length fragment of the core language is decidable.

Proof. Since $i_{1}, \ldots, i_{n}$ are integer constants, every value assignment that satisfies the formula assigns to $s_{k}$ a string of length $i_{k}$, for all $k=1 \ldots n$. There exists only a finite number of value assignments with this property, so by substituting these value assignments the formula can be replaced by a finite disjunction of formulas with no free variables. It remains to show that satisfiability of closed formulas of the core language is decidable. This can be proved by induction on the depth of quantifiers in a formula $F$. If the number of quantifiers in $F$ is $0, F$ is a variable-free quantifier-free formula which can simply be evaluated. Suppose now that $F$ has at least one quantifier, we will then show how to eliminate a quantifier in $F$. Take any quantified subformula $G$ of $F$ that is not in the scope of another quantifier. Without loss of generality we can assume that $G$ is of the form $\left(\forall i \in\left[e_{1} \ldots e_{2}\right]\right) H(i)$. Then $e_{1}$ and $e_{2}$ are variable free, so they can be evaluated to concrete integer values $k_{1}$ and $k_{2}$, hence $G$ can be replaced by the conjunction $\bigwedge_{k_{1} \leq k \leq k_{2}} H(k)$ having a smaller quantifier depth.

Note that this proof works for the string library with any finite number of functions or predicates on characters, since for such functions we only need that they can be computed. Section 6 describes how our implementation uses the theory of arrays to delay relying on finite domains for solving constraints.

As a consequence of this theorem we obtain the following result. 
Theorem 2. The satisfiability problem for the fixed-length fragment of the library language without the function Replace is decidable.

Proof. Using definitions of Table 3 one can translate any formula of the library language without the function Replace into an equivalent formula of the core language. Since the translation does not introduce new free variables, any fixed-length formula is translated into a fixed-length formula. Then apply Theorem 1 on the decidability of the fixed-length fragment of the core language.

Theorem 2 and its proof provide a foundation for our method of constraint solving.

\section{Library Language Decidability and Undecidability Results}

In this section we consider the full (not fixed-length) library language. We will show that constraint solving for this language is undecidable. We will also point out that the decidability of a very large fragment of this language is equivalent to the decidability problem of a well-known problem related to word equations. Finally, we will prove some decidability results.

It is easy to see that several functions and predicates of the string library can be expressed using string concatenation. Among the representative subset selected by us these are Concat, Contains, Equals and StartsWith. Solving constraints using only such functions and predicates can be reduced to solving word equations and so is decidable. However, this fragment is hardly very practical.

Word Equations and Equal Length Constraints. Let us call the subset of $\mathcal{L} \mathcal{L}$ without Replace, ToUpper the pure library language. It is interesting that path feasibility in the pure library language is equivalent to a well-known extension of word equations whose decidability is an open problem. This problem is known as word equations with the equal length predicate. The equal length predicate, denoted by $\ell \ell$ is true on a pair of strings $s_{1}, s_{2}$ if and only if $s_{1}$ and $s_{2}$ have the same length. The decidability of word equations with the equal length predicate is an open problem [2]12].

Theorem 3. The path feasibility problem in the pure library language is decidable if and only if word equations with the equal length predicate are decidable.

Proof. We will show how to reduce the two problems to each other. To this end, we will first reformulate the constraint satisfaction problem for $\mathcal{L} \mathcal{L}$ as a problem on strings. To represent a character $c$ as a string we will use the string consisting of this character. To represent non-negative integers, we will use the following idea. Let us fix a letter of the alphabet, for example, $\mid$. We will use the string $|\ldots|$ of length $n$, denoted by $\widehat{n}$ as a representation for the number $n$ and call such strings numerals. To represent a negative number $-n$ we can use, for example, the string $-|\ldots|$ of length $n+1$, that is $-\widehat{n}$. Let us prove several facts about this representation. When we write that some relation can be represented we mean that it can be represented using an existential formula. Note the well-known fact [2] that inequations of words can be represented using equations, hence we will not consider negative literals in the proof.

Using word equations one can express that a string $s$ is a numeral. Indeed, consider the equation $s|=| s$. The solutions of this equation are exactly all numerals. Using 
word equations one can express the addition on integers. In our representation the addition on non-negative integers becomes string concatenation: indeed, $\widehat{m} \widehat{n}=\widehat{m+n}$ for all non-negative integers $m, n$. It is not hard to represent addition on all integers too. One can express the equal length predicate using the length function and vice versa. One direction is obvious since $\ell \ell\left(s_{1}, s_{2}\right) \equiv \ell\left(s_{1}\right)=\ell\left(s_{2}\right)$. In the other direction, note that the length of a string $s$ is equal to $n$ if and only if $s$ and $\widehat{n}$ have equal length. What remains to note is that for the pure library language functions both the equality and the inequality between these functions can be represented using string concatenation and the length function.

Let us now prove an undecidability result.

Theorem 4. The path feasibility problem for the library language is undecidable.

Proof. For every character $c$ of the alphabet consider the following function $\ell_{c}$ : for every string $s, \ell_{c}(s)=\widehat{n}$, where $n$ is the number of occurrences of $c$ in $n$. We will use the following result from [2]: the existential theory of words with concatenation, the equal length predicate and functions $\ell_{0}, \ell_{1}$ is undecidable. We already proved in Theorem 3 that the equal length predicate is expressible in the string library language, it remains to note that, for every character $c, \ell_{c}$ is expressible using the library function Replace.

One can prove other decidability results about automatic structures, we will only briefly sketch how one can prove them here. Let us call a predicate or a function on strings automatic if it can be represented by a finite automaton, for details see [10]. To apply this definition to integers and characters we assume that they are represented respectively as numerals and as one-character strings. The string library contains several automatic functions, namely Chars, Compare, Equals, Length, StartsWith, ToString, ToUpper. Other functions are not automatic but have automatic instances when one or more arguments are instantiated to constants. For example, for every constant string $s_{2}$, Concat $\left(s_{1}, s_{2}\right)$ considered as a function of $s_{1}$ is automatic. It is also automatic if we fix the first argument $s_{1}$ to be constant. It is known that the full first-order theory of every automatic structure (structure in which all predicates and functions are automatic) is decidable. However, this result is hardly interesting in practice for two reasons. Firstly, using automata-based method on large alphabets is prohibitively expensive. Secondly, the resulting decidable fragment is too narrow for applications, for example, it does not include concatenation and integer addition.

\section{Solving Constraint Satisfaction Problems in the Library Language}

Our algorithm for checking constraint satisfaction is described here and works as follows. First, we replace subterms by fresh variables to obtain a flattened constraint $C$. Then, produce a so-called integer abstraction of the problem. The integer abstraction is a quantifier-free formula $I$ of linear arithmetic over two kinds of integer variables: those coming from the constraint $C$ and those denoting the lengths of string variables occurring in $C$. After that we look for small solutions to $I$. Small solutions are obtained from 
Table 4. Integer abstraction of library predicates

\begin{tabular}{|c|c|}
\hline function & abstraction \\
\hline Compare $\left(s_{1}, s_{2}\right)=c$ & $\left(\ell\left(s_{1}\right)=0 \rightarrow c \leq 0\right) \wedge\left(\ell\left(s_{2}\right)=0 \rightarrow c \geq 0\right)$ \\
\hline $\operatorname{Concat}\left(s_{1}, s_{2}\right)=s$ & $\ell(s)=\ell\left(s_{1}\right)+\ell\left(s_{2}\right)$ \\
\hline $\operatorname{Contains}\left(s_{1}, s_{2}\right)$ & $\ell\left(s_{1}\right) \geq \ell\left(s_{2}\right)$ \\
\hline $\operatorname{IndexOf}_{4}\left(s_{1}, s_{2}, i_{1}\right)=i$ & $i=-1 \vee\left(i \geq i_{1} \wedge i+\ell\left(s_{2}\right) \leq \ell\left(s_{1}\right)\right)$ \\
\hline LastIndexOf $(s, c)=i$ & $i=-1 \vee i<\ell(s)$ \\
\hline Replace $_{2}\left(s_{1}, s_{2}, s_{3}\right)=s_{0}$ & $\begin{array}{l}\left(\ell\left(s_{2}\right) \geq \ell\left(s_{3}\right) \rightarrow \ell\left(s_{1}\right) \geq \ell\left(s_{0}\right)\right) \wedge \\
\left(\ell\left(s_{2}\right) \leq \ell\left(s_{3}\right) \rightarrow \ell\left(s_{1}\right) \leq \ell\left(s_{0}\right)\right)\end{array}$ \\
\hline $\operatorname{StartsWith}\left(s_{1}, s_{2}\right)$ & $\ell\left(s_{1}\right) \geq \ell\left(s_{2}\right)$ \\
\hline $\operatorname{Substring}_{1}\left(s_{1}, i\right)=s_{0}$ & $\ell\left(s_{0}\right)=\ell\left(s_{1}\right)-i$ \\
\hline Substring $_{2}\left(s_{1}, i, j\right)=s_{0}$ & $\ell\left(s_{0}\right)=j \wedge \ell\left(s_{1}\right) \geq i+j$ \\
\hline ToString $(c)=s$ & $\ell(s)=1$ \\
\hline ToUpper $\left(s_{1}\right)=s_{0}$ & $\ell\left(s_{0}\right)=\ell\left(s_{1}\right)$ \\
\hline
\end{tabular}

feasible solutions by imposing and repeatedly tightening bounds on string lengths. If there is no such solution, then the original constraint is unsatisfiable. If $I$ has a solution it gives us the lengths of all string variables in $C$. When we fix the length, we obtain a fixed-length formula in the core language which can be decided by a finite domain solver. If this formula has no solution, backtrack and try to find another solution of $I$.

Flattening. A constraint $C$ is called flat if all string library functions occur in $C$ at the top level, that is, for every term of formula $p\left(t_{1}, \ldots, t_{n}\right)$ occurring in $C$, where $p$ is different from equality, the terms $t_{1}, \ldots, t_{n}$ contain no occurrences of the string library functions. For example, the constraint Substring ${ }_{1}\left(s_{1}, i_{1}+\right.$ $\left.i_{2}\right)$ is flat while the constraint $s_{1}=$ Concat $\left(s_{2}\right.$, Substring $\left._{1}\left(s_{3}, 1\right)\right)$ is not, since Substring does not occur at the top level. Constraints with flat literals are created by introducing extra variables for subterms. For example, the constraint of $i_{1}=\operatorname{LastIndexO}_{1}\left(s_{1},,^{\prime} /^{\prime}\right)$. $\neg i_{1}<0$. $s_{2}=\operatorname{Substring}_{1}\left(s_{1}, i_{1}+1\right)$. Contains $\left(s_{2}\right.$, "EasyChair"). StartsWith $\left(s_{1}\right.$, "http: / / "). $i_{2}=\operatorname{Length}($ "http: / / ). $s_{3}=$ Substring $_{2}\left(s_{1}, i_{2}, i_{1}-i_{2}\right)$. $\operatorname{StartsWith}\left(s_{3}\right.$, "www. " ). $i_{3}=$ Length( "www. " ). $s_{4}=\operatorname{Substring}_{1}\left(s_{3}, i_{3}\right)$. $s_{4}=$ "live.com" Example 2 will become as shown on the right.

Integer abstraction. The integer abstraction of a literal defines necessary conditions for the literal to be true. This implies that every solution to the literal must also be a solution to the integer abstraction. For every literal $L$ in a flat constraint its integer abstraction is built as follows. First, let $F^{e}$ be the exception condition corresponding to the literal $L$ in Table 2, $F^{e}$ is false if there are no matching exception conditions for $L$. If the literal $L$ matches an entry in Table 4 with formula $F^{i}$, then the integer abstraction of $L$ is given as $\neg F^{e} \wedge F^{i}$. If $L$ is a negation $\neg L^{\prime}$ and $L^{\prime}$ matches an entry in Table 4 then the abstraction is given as $\neg F^{e}$; otherwise, the abstraction of $L$ is set to $L$. The flat constraint for our example has the integer abstraction given in Figure 2 . 


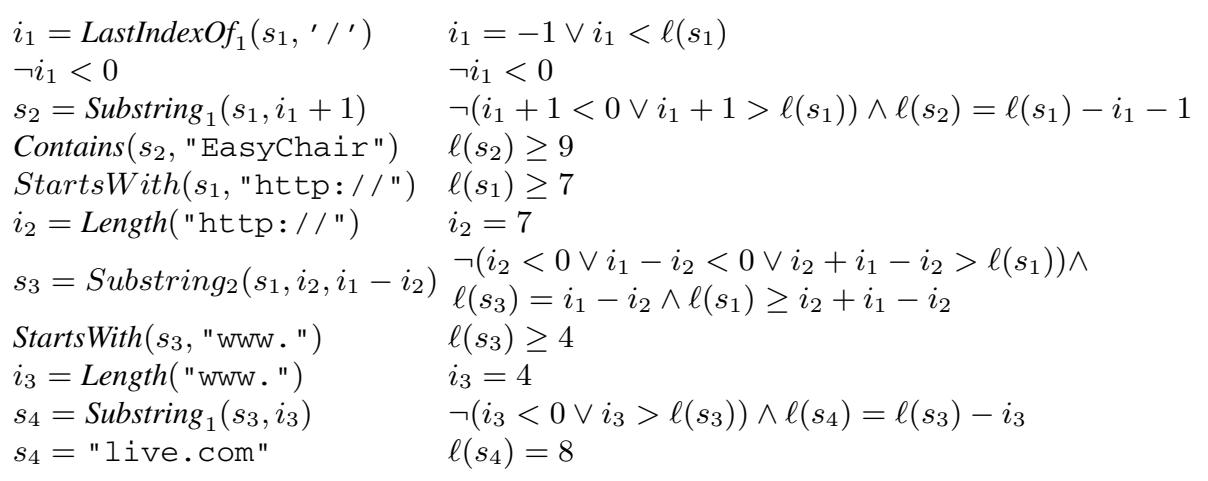

Fig. 2. Integer abstraction of the example program

Fixed-length constraint satisfaction problems. A constraint $C$ is said to be fixedlength if for every string variable $s$ occurring in $C$, the constraint also contains a literal of the form $\ell(s)=i$, where $i$ is an integer constant. One can easily note that in this case for every solution of $C$ the length of $s$ is $i$.

Consider any flat constraint $C$ and its integer abstraction $I$. If $I$ is unsolvable, then $C$ has no solution. Let us now take any value assignment $v$ that solves $I$ and consider the constraint $C^{\prime}$ obtained by adding to $C$ all constraints of the form $\ell(s)=v(i)$, where $s$ is a string variable occurring in $C$ and $i$ the integer variable denoting the length of $s$. One can immediately see that $C^{\prime}$ is a fixed-length constraint and that every solution to $C^{\prime}$ is also a solution to $C$. Our next observation is that the satisfiability problem for fixed-length constraints is decidable by Theorem 1 .

\section{Implementation in Pex and Integration with the SMT Solver Z3}

In this Section we describe how string library functions and symbolic string constraints are represented. We then describe how the Pex [16] tool produces symbolic string constraints. We observe that several of the string library functions can be handled using two functions we call Shift and Fuse. Finally, we describe using the SMT solver Z3 for solving string constraints. We experiment with different strategies for the integration.

In Pex, Strings are represented as an abstract type String. We use one predicate and two functions for accessing strings. The predicate null $(s)$ is true if the string $s$ is a null pointer. The function length $(s)$ results in the length of $s$, and the function chars $(s)$ results in an array, whose domain consists of 32-bit bit-vectors and the range is the set of unicode characters (16-bit bit-vectors).

Building abstract execution paths. All string library functions have implementations in Microsoft's .NET base class library, but many of these are in native code and therefore not in the scope of what Pex can analyze (Pex only analyzes .NET code). Pex therefore contains straightforward implementations of each of the string library functions written in C\#. We show the implementation of the IndexOf function as an example below. 


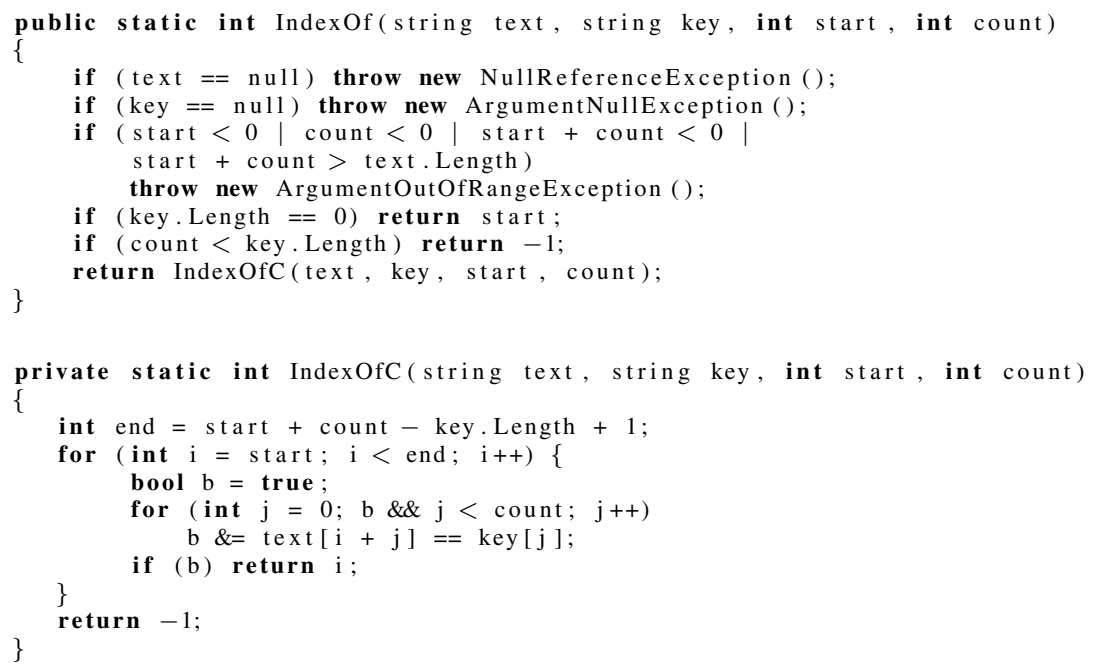

The implementation contains two parts, the preamble within the body of IndexOf is a straight-line code sequence that checks for exception conditions and boundary values. These checks include the conditions listed in Table 2 and parts from the abstraction of Table 4 that cover also the concrete case. Then, the portion of the string function that we wish to abstract is encoded in IndexOfC.

At this point we can run standard dynamic symbolic execution with the string library functions by using the instructions within IndexOf and IndexOfC for both the concrete and symbolic execution. Our aim is however to abstract the part of IndexOfC into core string constraints. For this purpose we introduce the uninterpreted function IndexOfA. When executing IndexOfC in dynamic symbolic execution, we do not add constraints to the path condition, but set the symbolic result to IndexOfA (text, key, start, count).

Functions, such as Concat and Substring, do not depend on the string tokens. Pex encodes such functions using two primitives, Shift and Fuse, axiomatized by $\operatorname{Shift}(a, i)[j] \simeq a[i+j]$ and $\operatorname{Fuse}(a, i, b)[j] \simeq$ if $j<i$ then $a[j]$ else $b[j]$. We can then replace chars $(\operatorname{ConcatA}(a, b))$ by $\operatorname{Fuse}(\operatorname{chars}(a)$, length $(a), \operatorname{Shift}(\operatorname{chars}(b)$, -length $(a)))$, and chars(SubstringA $(a, i, j))$ by Shift $(\operatorname{chars}(a), i)$.

Solving string constraints. For each execution path we get a path condition and perform multiple queries to $\mathrm{Z} 3$ using the algorithm outlined next. It uses constants $\mathcal{U}$ to bound the length of strings, $\mathcal{N}$ to bound the number of calls to Z3. In our implementation, both $\mathcal{U}$ and $\mathcal{N}$ are used to bound the search.

Phase 1. Assert the path condition $\pi$, the axioms for Fuse and Shift, and the axiom $\forall s .0 \leq \operatorname{length}(s)<\mathcal{U}$, where $\mathcal{U}$ is a fresh constant. If the constraints are unsatisfiable, then fail; otherwise enter the second phase.

Phase 2. We are given a path constraint $\pi$ that is satisfiable with respect to partial unfoldings of the supplied axioms. Find the smallest power of two for $\mathcal{U}$ such that the constraints have a model. Set $\mathcal{N} \leftarrow 0$.

1. Extract values from the model that suffice to create a finite unfolding of all quantifiers used in Table 3 for the functions: IndexOf, LastIndexOf, 
Contains, Compare, and Equals. Thus, we assert the definitions of these functions replacing all quantifiers of the form $(\forall i \in[a \ldots b]) \varphi(i)$ with a finite set of assertions $\varphi(v(a)), \ldots, \varphi(v(b))$.

2. Instantiate the definitions of Shift and Fuse1.

3. If the constraints are satisfiable, return the current model.

4. Fail if $\mathcal{N}$ or $\mathcal{U}$ exceed pre-configured bounds.

5. Otherwise, undo the assertions from steps 1 and 2 and force a solution with increased lengths by asserting $\Sigma_{i} \ell\left(s_{i}\right)>\Sigma_{i} v\left(\ell\left(s_{i}\right)\right)$ where $\ell\left(s_{i}\right)$ occur in $\pi$.

6. If the new constraints are unsatisfiable, then fail.

7. Otherwise, repeat step 1 with $\mathcal{U} \leftarrow 2 \cdot \mathcal{U}, \mathcal{N} \leftarrow \mathcal{N}+1$,

Note that step 5 can prevent exploring models where string lengths add up to the previous value, but are re-distributed in a different way. While $\mathcal{N}$ and $\mathcal{U}$ impose limits on which models are explored, it is the case that our implementation in Pex makes implicit use of properties of the bit-vector solver in Z3 in the folowing way: Z3 has a preference for models of bit-vectors where the most significant bits are set, so the progression of lengths tends to grow in proportion to $\mathcal{U}$.

To distribute length increases fairly among strings, Pex furthermore excludes strings whose length was increased recently, unless this exclusion would cause the constraints to become unsatisfiable.

\section{Experiments}

We applied Pex on the EasyChain query given in Figure 1 using different search strategies to flip branches of already discovered execution paths (which includes unrolling loops): breadth-first (BFS), depth-first (DFS), flipping of Random branches, and Pex' default strategy [17] (which combines several heuristics). We compare those strategies with a partial implementation of the algorithm described above, where only Concat, Substring, Remove and Insert are abstracted, and the fully abstract version of the algorithm. We set the Table 5. Evaluation of string solver on EasyChair

\begin{tabular}{|l|r|r|}
\hline mode & time/s & paths \\
\hline \hline BFS & 7.90 & 214 \\
\hline DFS & 3.65 & 51 \\
\hline Random & 8.73 & 196 \\
\hline Default & 0.83 & 35 \\
\hline Partial & 0.61 & 30 \\
\hline Abstract & 1.02 & 19 \\
\hline
\end{tabular}
bounds of $\mathcal{U}$ at $2^{12}$, and $\mathcal{N}$ at 3 . Table 5 shows the results: BFS, DFS and Random search performed clearly worse than the default and abstract strategies. The partial abstraction resulted in the fastest run-time and fewer explored paths than the default exploration strategy, while complete abstraction required exploring fewer paths, but took slightly more overall time.

A different example that highlights the effectiveness of the abstraction can be constructed by creating a program test with a conjunction of the form $s \cdot \operatorname{IndexOf}\left(s_{1}\right) !=-$ $1 \& \& s . \operatorname{IndexOf}\left(s_{2}\right) !=-1 \& \& \ldots$, where $s_{1}, s_{2}, \ldots, s_{c}$ are different non-overlapping strings with a long common prefix. The goal is to synthesize a string $s$ that contains all the substrings $s_{1}, s_{2}, \ldots s_{c}$. Table 6 summarizes the results of trying a progression of

\footnotetext{
${ }^{1}$ While necessary for completeness, this step has not had any effect in our experiments.

${ }^{2}$ All experiments were performed with a Intel Core 2 CPU T7400 @ 2.16 Ghz, 4GB RAM.
} 
Table 6. Evaluation of string solver on IndexOf progression

\begin{tabular}{|c|c|c|c|c|c|c|c|c|c|c|c|c|}
\hline$c$ & \multicolumn{2}{|r|}{1} & \multicolumn{2}{|c|}{2} & \multicolumn{2}{|c|}{3} & \multicolumn{2}{|c|}{4} & \multicolumn{2}{|c|}{5} & \multicolumn{2}{|l|}{6} \\
\hline mode & time & paths & time & paths & time & paths & time & paths & time & baths & time & paths \\
\hline BFS & 0.14 & 5 & 4.50 & 140 & 73.34 & 1432 & 960.08 & 7727 & time & & times & \\
\hline Random & 0.26 & 7 & 0.42 & 9 & 4.93 & 57 & 16.70 & 108 & 40.55 & 199 & 154.36 & 541 \\
\hline Default & 0.23 & 6 & 0.62 & 14 & 7.03 & $\overline{78}$ & 7.76 & 80 & 8.81 & $\overline{82}$ & \begin{tabular}{|l|}
193.49 \\
\end{tabular} & 637 \\
\hline Abstract & 0.15 & 6 & 0.33 & 8 & 0.99 & 10 & 2.02 & 12 & 4.44 & 14 & 6.67 & 16 \\
\hline
\end{tabular}

$c=1, \ldots, 6$ such conjuncts. DFS search, not shown, does not even manage to explore a single path, other strategies are also inferior to abstraction.

\section{Conclusion}

We presented a two-tier approach for generating finite models of path constraints for string-manipulating programs. Our approach views constraints from string libraries as extensions of the word equation problem and we identified decidable, undecidable, and open problems in the context of word equations. Our approach was integrated with the dynamic symbolic execution engine Pex.

Related Work. In the context of symbolic execution of programs, string abstractions has been recently studied by Ruan et.al. [13], where an approach based on a first-order encoding of string functions is proposed. They study $\mathrm{C}$ programs where strings are zero-terminated arrays whose lengths are bounded by constants. The first-order quantifiers can therefore be finitely unfolded and decided using a solver for linear arithmetic and assignments. Ruan et.al. [5] also fix length of strings in order to obtain a decidable fragment, but don't consider increasing the size of strings in the search of models. Shannon et.al. [15] use automata-based representations for abstracting strings during symbolic execution of Java programs. They handle a few core methods in the java.lang.String class, and some other related classes. They integrate a numeric constraint solver, but apparently in a partial way. For example, string methods which return integers, such as IndexOf, cause case-splits over all possible return values within certain bounds. Automata-based methods have been pursued in the context of static analysis by Christensen et.al. [4], where automata, using the Mohri-Nederhof algorithm, represent over-approximations of possible string values. A motivation for the work is SQL injection attacks. The same motivation also inspired Fu et.al. [6]. They first solve Boolean and integer constraints to obtain a model and then proceed to solving string constraints by using the obtained model to build automata for the string constraints. To our knowledge, the mentioned automata-based methods require case analysis outside of their calls to their constraint solvers and automaton construction phases. In our framework, case analysis is integrated with the constraint solver pass.

Future work. A plethora of future work is possible in the context of exploring string manipulating programs. Regular expressions are often used by the discriminating programmer to accomplish string manipulation. In particular, our running EasyChair example can be directly encoded using a regular expression. But real regular expression 
libraries can encode side-effects and non-regular properties. Can such extensions be handled by methods presented here? A different direction is to extend array property fragments [19] to handle common string queries. We would also like to use information encoded in the core language to control the constraint solver programmatically. For example, one could alternate quantifier unfolding with solving for the bounds.

Acknowledgments. We thank the referees for detailed and constructive feedback, Wolfram Schulte for numerous early stage discussions and Yuri Matiyasevich for his help on finding related work on word equations.

\section{References}

1. Bradley, A.R., Manna, Z., Sipma, H.B.: What's decidable about arrays? In: Emerson, E.A., Namjoshi, K.S. (eds.) VMCAI 2006. LNCS, vol. 3855, pp. 427-442. Springer, Heidelberg (2005)

2. Büchi, J.R., Senger, S.: Definability in the existential theory of concatenation. Zeitschrift fur Mathematische Logik und Grundlagen der Mathematik (1988)

3. Cadar, C., Ganesh, V., Pawlowski, P.M., Dill, D.L., Engler, D.R.: Exe: automatically generating inputs of death. In: CCS, pp. 322-335. ACM Press, New York (2006)

4. Christensen, A.S., Møller, A., Schwartzbach, M.I.: Precise analysis of string expressions. In: Cousot, R. (ed.) SAS 2003. LNCS, vol. 2694, pp. 1-18. Springer, Heidelberg (2003)

5. Dong, Y., Quan, Q., Zhang, J.: Priority-based energy aware and coverage preserving routing for wireless sensor network. In: VTC Spring, pp. 138-142. IEEE, Los Alamitos (2008)

6. Fu, X., Lu, X., Peltsverger, B., Chen, S., Qian, K., Tao, L.: A Static Analysis Framework For Detecting SQL Injection Vulnerabilities. In: COMPSAC, pp. 87-96 (2007)

7. Godefroid, P.: Compositional dynamic test generation. In: Proc. of POPL 2007, pp. 47-54. ACM Press, New York (2007)

8. Godefroid, P., Klarlund, N., Sen, K.: DART: directed automated random testing. SIGPLAN Notices 40(6), 213-223 (2005)

9. Habermehl, P., Iosif, R., Vojnar, T.: What else is decidable about integer arrays? In: Amadio, R. (ed.) FOSSACS 2008. LNCS, vol. 4962, pp. 474-489. Springer, Heidelberg (2008)

10. Khoussainov, B., Nies, A., Rubin, S., Stephan, F.: Automatic structures: Richness and limitations. In: LICS, pp. 44-53 (2004)

11. King, J.C.: Symbolic execution and program testing. Commun. ACM 19(7), 385-394 (1976)

12. Matiyasevich, Y.: Word Equations, Fibonacci Numbers, and Hilbert's Tenth problem. In: Workshop on Fibonacci Words, vol. 43, pp. 36-39 (2007)

13. Ruan, H., Zhang, J., Yan, J.: Test Data Generation for C Programs with String-Handling Functions. Theoretical Aspects of Software Engineering 0, 219-226 (2008)

14. Sen, K., Agha, G.A.: CUTE and jCUTE: Concolic unit testing and explicit path modelchecking tools. In: Ball, T., Jones, R.B. (eds.) CAV 2006. LNCS, vol. 4144, pp. 419-423. Springer, Heidelberg (2006)

15. Shannon, D., Hajra, S., Lee, A., Zhan, D., Khurshid, S.: Abstracting symbolic execution with string analysis. In: Taicpart-Mutation, Washington, DC, USA, pp. 13-22 (2007)

16. Tillmann, N., de Halleux, J.: Pex - white box test generation for .NET. In: Beckert, B., Hähnle, R. (eds.) TAP 2008. LNCS, vol. 4966, pp. 134-153. Springer, Heidelberg (2008)

17. Xie, T., Tillmann, N., de Halleux, P., Schulte, W.: Fitness-guided path exploration in dynamic symbolic execution. Technical Report MSR-TR-2008-123, Microsoft (2008) 